REVESCO. Revista de Estudios Cooperativos

ISSN: $1885-8031$

https://dx.doi.org/10.5209/REVE.69192

\title{
Burnout y work engagement: Demandas laborales, recursos y resultados organizativos en organizaciones de Acción Social
}

\author{
Rosa María Benítez Saña ${ }^{1}$ y Ana Rosa del Águila Obra ${ }^{2}[$ \\ Recibido: 15 de enero de 2020 / Aceptado: 3 de abril de 2020 / Publicado: 6 de octubre de 2020
}

Resumen. Esta investigación analiza dos procesos opuestos, coexistentes e interrelacionados, uno de deterioro de la salud del empleado, burnout, y otro motivador, work engagement. Desde el enfoque del modelo de Demandas y Recursos Laborales, se analizan las demandas laborales, antecedentes del burnout, y cómo se pueden modular sus efectos negativos, gracias a la potenciación de los recursos (laborales y personales). Esto es posible gracias, en parte, a la implementación por parte de las organizaciones de prácticas de recursos humanos de alto rendimiento. Estos recursos, a su vez, son antecedentes del work engagement del empleado, que influye positivamente en los resultados de la organización. Se ha empleado una combinación de métodos, tanto cuantitativos (tales como la escala UWES-9 y la MBI-GS), como cualitativos (basados en observación directa, focus group, entrevistas y encuesta STARs), en el ámbito de una organización de Acción Social (que atiende al colectivo de personas con discapacidad intelectual). Esto ha permitido constatar la existencia de correlaciones negativas entre el work engagement y las dimensiones que componen el burnout, e identificar que los profesionales de esta entidad, como informantes clave (empleados, directivos), perciben que el work engagement impacta positivamente en los resultados positivos organizacionales y cómo los sistemas de trabajo de alto rendimiento pueden actuar reduciendo las demandas laborales y potenciando los recursos, tanto laborales como personales, de los empleados. Estos hallazgos ponen de manifiesto la necesidad de prestar especial atención, en particular, a la selección, la formación y la participación, en el sistema de prácticas de recursos humanos en el Tercer Sector de Acción Social, dado su impacto tanto en el bienestar de los empleados como en los resultados organizacionales y sociales.

Palabras clave: Work engagement; Burnout; Prácticas de recursos humanos de alto rendimiento; Organizaciones de acción social; Discapacidad intelectual.

Claves Econlit: L300; L310; M540; D640.

\section{[en] Burnout and work engagement: Job Demands, resources and organizational outputs in Social Action organizations}

\begin{abstract}
This research analyzes two opposite, coexisting and interrelated processes, one of them the deterioration of the employee's health process (burnout), and the other one, a motivator process (work engagement). From the approach of the Job Demand Resources Model, labor demands, as antecedents of burnout, are analyzed, and how its negative effects can be modulated, thanks to the empowerment of resources (job and personal). This is made possible, in part, by organizations implementing high-performance human resource practices. These resources, in turn, are antecedents of the employee's work engagement, which positively influences the organization's results. A combination of methods, both quantitative, such as the UWES-9 scale and the MBI-GS, as well as qualitative, based on direct observation, focus group, interviews and STAR survey, has been applied. The study of a Social Action organization, which serves the group of people with intellectual disabilities, has confirmed the existence of negative correlations between work engagement and the burnout dimensions, and identified that the professionals of this entity, as key informants (employees, managers), perceive that work engagement positively impacts organizational positive results and how highperformance work systems can act by reducing job demands and empowering employees' resources, both professional and personal. These findings highlight the need to pay special attention, in particular, to the selection, training, and participation in the system of human resource practices in Social Action organizations, given its impact on both the well-being of employees and the organizational and social results.
\end{abstract}

Keywords: Work engagement; Burnout; Human resources practices; Social Action organizations; Intellectual disability.

Sumario. 1. Introducción. 2. Revisión de la literatura 3. Contexto de la investigación. 4. Metodología. 5. Resultados. 6. Discusión. 7. Conclusiones. 8. Referencias bibliográfícas. Anexo.

1 Universidad de Málaga, España.

Dirección de correo electrónico: rosabenitez@uma.es

2 Universidad de Málaga, España.

Dirección de correo electrónico: anarosa@uma.es. 
Cómo citar. Benítez Saña, R.M.; del Águila Obra, A.R. (2020) Burnout y work engagement: Demandas laborales, recursos y resultados organizativos en organizaciones de Acción Social. REVESCO. Revista de Estudios Cooperativos, vol. 136, e69192. https://dx.doi.org/10.5209/reve.69192.

\section{Introducción}

Según el último estudio publicado en España, actualmente operan unas 27.000 entidades en el Tercer Sector de Acción Social (TSAS), con un volumen de negocio que representa el 1,45 por ciento del Producto Interior Bruto (PIB, referido a 2018). Este conjunto de entidades emplea a unos 527.000 profesionales, lo que supone un 3 por ciento del empleo generado en el país, y pone de manifiesto la relevancia de dichas organizaciones en la economía española (POAS, 2019). Este desarrollo del TSAS está conectado además con tendencias a nivel europeo, la demanda cada vez mayor, los cambios legislativos, los desajustes en la financiación para la prestación de servicios, entre otras causas, han ido orientando a estas entidades a una imperativa profesionalización. Además, se evidencia una mayor participación de las mismas en la economía social (POAS, 2017).

Por otra parte, es destacable que en la actualidad existe una llamada a que las entidades y empresas sean responsables y transparentes, donde se promueva la igualdad, la inclusión y la igualdad de oportunidades, entre otros, a través, por ejemplo, de los Objetivos de Desarrollo Sostenible (ODS). Y se puede afirmar que las entidades del TSAS y de la Economía Social desde su propia base, sus valores y principios, ya se viene respondiendo con un modelo de gestión que persigue un desarrollo social sostenible y el logro de beneficios para el conjunto de la sociedad (Comos, 2018).

En este sentido, en su momento, la Estrategia Europa 2020 (European Commission, 2016), para un crecimiento inteligente, sostenible e integrador, estableció orientaciones para las políticas económicas de los Estados miembros y, entre otras cuestiones, recomendó la potenciación de este tipo de entidades con el objetivo de favorecer el desarrollo económico y social, planteándolas como un modelo alternativo de empresa, con capacidad de ser viables, competitivas y generadoras de valor económico y sobre todo social, lo que conlleva unos servicios profesionalizados, basados en calidad-excelencia.

Sin embargo, pese a su relevancia, tanto económica como en cuanto a la labor que desarrollan, son escasas las investigaciones sobre las entidades del TSAS, y más aún en lo relativo al modelo de gestión que siguen. En el ámbito concreto de las prácticas de gestión de personas, investigaciones previas, llevadas a cabo por diversos autores (Durán, Rey y Extremera, 2004; Extremera, Durán y Rey, 2005; Durán y Manteca, 2013), se ocuparon de analizar las condiciones laborales y las características personales de los empleados de entidades de Acción Social, a través de la medición de los niveles de burnout, satisfacción laboral y vital y el engagement, desde la perspectiva del modelo de Demandas y Recursos Laborales (modelo DRL). Los resultados pusieron de manifiesto el amplio potencial que presenta el work engagement (en adelante WE) como ventaja competitiva en este tipo de organizaciones.

Si bien, el estudio que del WE se ha desarrollado en el ámbito de las organizaciones sin ánimo de lucro, en el contexto internacional, aún es escasa (Park, Kim, Park, y Lim, 2018), y en menor medida se ha relacionado este constructo con la gestión de los recursos humanos como factor clave para lograr una prestación de servicios de calidad. Las entidades del TSAS necesitan desarrollar estrategias innovadoras en el ámbito de la gestión de personas para logar resultados organizacionales positivos, tales como mejoras en la calidad del servicio y el bienestar de sus empleados. Luego las investigaciones dirigidas a dar a conocer buenas prácticas en el ámbito de la Acción Social son relevantes, tanto desde el punto de vista académico como de gestión. Y es que los directivos de estas organizaciones deben llevar a cabo prácticas que minimicen el burnout de los empleados y que maximicen el compromiso de los mismos.

En este contexto, el propósito de investigación de este trabajo es el siguiente: Es posible potenciar el WE de los empleados en las entidades del TSAS a través de la implementación de sistemas de trabajo de alto rendimiento (en adelante STAR) para lograr mejorar la calidad del servicio y mejores resultados organizacionales y sociales. Y se definen los siguientes objetivos: 1) Proponer un rediseño del modelo DRL, incorporando las STAR; 2) Medir los niveles de burnout y WE de los profesionales de la Acción Social; 3) Analizar las correlaciones entre los constructos burnout y WE; 4) Conocer las percepciones de los profesionales de las entidades del TSAS sobre las demandas y recursos laborales, así como de los recursos personales, y su relación con las STAR y los resultados de la organización.

Para el logro de estos objetivos se ha desarrollado una metodología soportada en varios métodos, tanto cuantitativos como cualitativos. A través del estudio de una entidad del TSAS, que atiende a personas con discapacidad intelectual, se ha podido constatar la existencia de correlaciones negativas entre el WE y las dimensiones que componen el burnout, e identificar que los profesionales de esta entidad perciben que el WE impacta positivamente en los resultados positivos organizacionales y las STAR pueden actuar reduciendo las demandas laborales y potenciando los recursos tanto laborales como personales de los 
empleados. Estos hallazgos permiten arrojar recomendaciones para la gestión del capital humano de estas entidades.

\section{Revisión de la literatura}

Con el objeto de presentar un resumen del marco teórico de la investigación, es preciso considerar los conceptos relacionados, tales como el burnout, el WE, el modelo DRL y las STAR.

\subsection{Burnout}

El burnout ha sido objeto de numerosas investigaciones, y es considerado como uno de los riesgos psicosociales más extendidos a nivel mundial, constituyendo, incluso, una problemática de carácter transcultural (Salanova y Llorens, 2008). Las investigaciones de Maslach y Jackson (1986) lo describen como un síndrome que conlleva síntomas de agotamiento emocional, despersonalización y baja realización personal, y puede aparecer principalmente en profesionales que trabajan con personas y se implican en los problemas de éstas. Estos tres síntomas fueron identificados como las tres dimensiones básicas del síndrome de burnout. El agotamiento se define como una experiencia en la que los trabajadores sienten que no pueden dar más de sí mismos, debido a una sobrecarga en las tareas o las demandas laborales y tienen la sensación de que la fuerza, o capital emocional de que disponen, se va consumiendo. El cinismo, por su parte, se caracteriza por el desarrollo por parte del trabajador de actitudes y conductas negativas hacia las personas o usuarios, que reciben en el servicio que prestan (por ejemplo, personas con discapacidad intelectual que reciben los cuidados del trabajador en un centro ocupacional o residencia). La baja eficiencia profesional, es la sensación del empleado de no tener la habilidad o capacidad suficiente para realizar su trabajo, lo que lo lleva a evaluarse negativamente, sintiéndose infeliz y descontento consigo mismo y con su labor (Maslach y Leiter, 2001; Maslach, Schaufeli y Leiter, 2001).

El burnout es considerado un estado mental persistente y negativo que experimenta el empleado, y que se refleja directamente en una disminución del rendimiento y reducción de la calidad del desempeño del trabajo, ya que el trabajador no se siente capaz de realizarlo adecuadamente (Schaufeli y Enzmann, 1998). Se han llevado a cabo investigaciones a nivel de empleos relacionados con la prestación directa de servicios, donde se observa su manifestación cuando las demandas exceden los recursos de los empleados (Peiró, 2004). En relación con los desencadenantes del burnout, y al objeto de proteger a los trabajadores de la exposición a condiciones de trabajo que entrañen este riesgo, según señala Fidalgo (2006a), se han de considerar diversos grupos de estresores a nivel de organización (estructura organizativa muy jerarquizada, falta de apoyo instrumental por parte de la organización, exceso de burocracia, falta de participación, falta de formación, falta de desarrollo profesional, estilo de dirección inadecuado, desigualdad percibida en la gestión de personas, etc.), a nivel del diseño de puesto de trabajo (sobrecarga de trabajo, falta de tiempo para atención al usuario o paciente, falta de apoyo social, poca autonomía, disfunciones de rol, carga emocional excesiva, etc.) y los relativos a las relaciones interpersonales (relaciones conflictivas con clientes/pacientes/usuarios, relaciones, conflictos entre compañeros y con usuarios, ausencia de reciprocidad en los intercambios sociales, etc.).

En suma, se puede afirmar que el burnout hace referencia a un daño psicosocial, que afecta a la salud psíquica de los trabajadores, cuyo origen se localiza en las condiciones laborales derivadas de la organización del trabajo, y que sucede como consecuencia de la exposición a estresores laborales debidos a una sobrecarga en las demandas y/o la carga de trabajo, que provocan cansancio emocional, debido a la insuficiencia de los recursos de que disponen los trabajadores para afrontarlas con éxito, lo que genera finalmente una respuesta defensiva traducida en despersonalización o cinismo frente a la tarea y las personas para las que se trabaja, y que provoca un sentimiento de baja autorrealización del trabajador, unida a sentimientos de baja eficacia profesional. En cuanto al impacto del burnout, en la organización los síntomas se manifiestan en un deterioro de la comunicación y de las relaciones interpersonales, disminuyen el compromiso del empleado y el rendimiento laboral. Lo anterior puede traducirse en un mayor índice de absentismo, rotación y abandono, en general, se pueden ver afectadas la calidad del servicio y la satisfacción de los clientes (Fidalgo, 2006b).

\subsection{Work engagement}

En 1990, Kahn introduce dos conceptos opuestos, engagement personal y disengagement personal, y esto supone que la persona se define a sí misma en relación con el trabajo que realiza y en qué organización lo realiza, aproximándonos al sentimiento de orgullo de las personas por su profesión y la organización a la que pertenecen (Kahn, 1990). Por su parte, Maslach y otros (2001) conceptualizaron el WE como la antítesis positiva del burnout. 
Pese a contar con unas tres décadas de historia, el WE es un constructo considerado relativamente novedoso, que ha sido estudiado en primer lugar desde el prisma de la Psicología de la Salud Ocupacional Positiva (PSOP), la cual pone su enfoque en las fortalezas humanas y el funcionamiento óptimo del ser humano (Luthans, Avolio, Avey y Norman, 2007; Salanova, Martínez y Llorens, 2005; Schaufeli y Bakker, 2003; Seligman y Csikszentmihalyi, 2014). Posteriormente, se han realizado estudios desde la perspectiva de la Dirección de Empresas (Barrick, Thurgood, Smith y Courtright, 2015), donde se ha puesto de manifiesto que, con el diseño motivador de puestos, las prácticas de gestión de personas adecuadas y el liderazgo transformacional de los equipos directivos el WE puede ser gestionado por las organizaciones, siendo considerado un constructo presente en la organización, no solo a nivel individual, de manera estratégica puede potenciarse a nivel colectivo.

El WE se define como "un estado mental positivo relacionado con el trabajo y caracterizado por vigor, dedicación y absorción. Más que un estado específico y momentáneo, el engagement se refiere a un estado afectivo-cognitivo que no está focalizado en un objeto, evento o situación particular" (Schaufeli, Salanova, González-Romá y Bakker, 2002:74). La dimensión vigor se caracteriza por "altos niveles de energía y resistencia mental mientras se trabaja, el deseo de esforzarse en el trabajo que se está realizando incluso cuando se presentan dificultades en el camino". La dedicación denota la "alta implicación laboral, junto con la manifestación de un sentimiento de significación, entusiasmo, inspiración, orgullo y reto por el trabajo" que experimentan los empleados. Este sentimiento de significación les hace identificarse fuertemente con su trabajo, lo que también se puede relacionar con estar orgulloso del trabajo que se realiza, además de sentirse entusiasmado por el desafío que supone su desempeño. Finalmente, la absorción tiene lugar "cuando se está totalmente concentrado en el trabajo, mientras se experimenta que el tiempo «pasa volando» y se tienen dificultades para desconectar de lo que se está haciendo debido a fuertes dosis de disfrute y concentración experimentadas" (Schaufeli et al, 2002:74-75).

En resumen, se puede decir que el WE es un constructo que se desarrolla en tres dimensiones: vigor, dedicación y absorción, y que puede considerarse un estado mental positivo y persistente en el que se encuentra el empleado mientras realiza su trabajo. El empleado, durante su jornada laboral se encuentra feliz, lleno de energía y empuje para llevar a cabo sus tareas (vigor). Posee además un fuerte vínculo emocional positivo hacia su trabajo del que se siente orgulloso porque le entusiasma, lo encuentra lleno de significado y supone además un desafío y fuente de inspiración para él (dedicación). Finalmente, el empleado tiene la sensación de que durante la realización de su trabajo el tiempo "pasa volando" y éste le resulta tan gratificante que incluso le cuesta abandonar la tarea en la que se encuentra altamente concentrado (absorción). En definitiva, el empleado siente verdadera pasión por su trabajo por lo que no le cuesta invertir esfuerzo extra en realizarlo.

\subsection{Modelo DRL}

El modelo DRL se identifica como una de las primeras aproximaciones teóricas realizadas con objeto de comprender los antecedentes del burnout (Demerouti, Bakker, Nachreiner y Schaufeli, 2001). Es gracias a esta investigación como comienzan a surgir los primeros estudios sobre WE, centrándose en los aspectos positivos considerados moduladores del burnout. En el modelo DRL (Demerouti et al, 2001; Bakker y Demerouti, 2008; Salanova y Schaufeli, 2009), confluyen dos procesos opuestos pero coexistentes e interrelacionados: un proceso de deterioro de la salud y otro proceso motivador. El primer proceso está caracterizado por el burnout, que ejerce importantes efectos negativos tanto en los empleados (absentismo, errores, quejas psicosomáticas) como en la organización (mal clima laboral, malos resultados organizacionales). El segundo proceso está caracterizado por el WE y sus consecuencias positivas implican una serie de ventajas para el empleado y la organización. Por un lado, los empleados con WE se muestran más motivados, presentan niveles altos de satisfacción laboral, menores índices de absentismo, y se encuentran más vinculados con la empresa, lo que genera una espiral de ganancias mutuas, que se traduce en mejores resultados económicos y sociales.

El modelo teórico DRL identifica una serie de demandas y recursos, determinantes del nivel de desempeño en las tareas de los empleados. Las demandas laborales, que subyacen tras el burnout, son definidas como "aquellos aspectos del trabajo, organizacionales, físicos o sociales, que requieren un esfuerzo físico y mental y que además llevan asociados costes físicos y psicológicos" (Demerouti et al, 2001:501). Entre las demandas laborales se incluyen (Schaufeli y Taris, 2014): complejidad de la tarea, inseguridad laboral, demandas del desempeño, cambios desfavorables de horarios, sobrecarga cualitativa de trabajo, riesgos laborales, etc. Asimismo, los recursos laborales fueron definidos como "aquellos aspectos físicos, sociales u organizacionales del trabajo que hacen posible alguno de los siguientes aspectos: ser funcionales al alcanzar los objetivos laborales, reducir las demandas laborales y los costes fisiológicos y psicológicos asociados, estimular el WE dentro de las mismas, favorecer el desarrollo y crecimiento personal" (Demerouti et al, 2001:501). Entre los principales recursos laborales destacan: desarrollo profesional, autonomía, 
creatividad, recompensas económicas, clima de innovación, reto laboral, participación, etc. (Schaufeli y Taris, 2014)

Aunque en principio se pensó que las demandas laborales se afrontaban únicamente con los recursos laborales, posteriormente se llevó a cabo una nueva revisión que se ocupó de incorporar al modelo DRL otro tercer factor, los recursos personales (Bakker y Demerouti, 2008), que completan este marco teórico, y son definidos como "aquellas características positivas de las personas, que tienen la capacidad de reducir o amortiguar el potencial impacto negativo de las demandas pero que a la vez pueden ser origen por si mismos de procesos positivos como el crecimiento personal y profesional" (Salanova y Schaufeli, 2009:149). Además, fue incluido el capital psicológico (CapPsic) como integrante de los recursos personales, considerándolo como un importante factor determinante de WE (Bakker y Demerouti, 2008). El CapPsic se compone de cuatro dimensiones (Luthans, Luthans y Luthans 2004; Luthans et al, 2007): autoeficacia (confianza que tiene el empleado en las propias capacidades), esperanza (voluntad o confianza en que se lograrán los objetivos deseados), resiliencia (capacidad para superar circunstancias adversas) y optimismo (tendencia a ver y juzgar las cosas de forma positiva). En esa misma línea, estudios más recientes, analizan diversas organizaciones y se muestra cómo sus líderes, a través de la potenciación del CapPsic, consiguen fomentar mayores niveles de engagement en los empleados ejerciendo a su vez un impacto positivo en la mejora de los resultados de la organización (Thompson, Lemmon y Walter, 2015).

\subsection{Sistemas de trabajo de alto rendimiento}

Determinados estudios, examinan el impacto de las prácticas de gestión de personas, entre otros, en la productividad laboral y en el WE. Sus conclusiones sugieren que a través de la implementación de las adecuadas prácticas de gestión de personas se incrementa el nivel de WE de los empleados, debido a que se potencian los recursos laborales y personales de los empleados (Van De Voorde, Veld y Van Veldhoven, 2016). Estos estudios vienen a completar otros (Salanova y Schaufeli, 2009), que han venido considerando clave tener en cuenta, que las organizaciones obtienen mejores resultados a través de la utilización de prácticas de recursos humanos de alto rendimiento, como facilitadoras de WE.

Entre estas prácticas destaca, por ejemplo, el modelo de Organizaciones Saludables (Healthy and Resilient Organization-HERO), en boga hoy en día, como modelo de gestión para mejorar el bienestar de los empleados. Este modelo describe una combinación de tres principales componentes (Acosta et al, 2011; Salanova et al, 2013; Llorens, Salanova, Torrente y Acosta, 2013): 1) recursos y prácticas organizacionales saludables (soporte social, estrategias familia-trabajo, liderazgo transformacional); 2) equipos de empleados saludables (confianza organizacional) y 3) resultados organizacionales saludables (alto desempeño, responsabilidad social corporativa).

Asimismo, son también de interés las STAR, cuyo modelo ha sido adaptado por distintos autores (Arocas y Torres, 2006; Lertxundi y Landeta, 2012; Stirpe y Revilla, 2013; Tang y Tang, 2012). En este modelo se incluyen intervenciones organizacionales en áreas como: políticas de selección, formación, evaluación del desempeño, políticas retributivas, participación, comunicación, empowerment, conciliación de vida familiar, estabilidad laboral y relaciones interpersonales. Estas prácticas STAR tienen un impacto positivo en la generación de recursos laborales y personales y, de esta manera, es posible deducir que los empleados, mediante el uso de estos recursos, pueden ser capaces de amortiguar el impacto negativo de las demandas laborales sobre la salud (presente en forma de burnout), y a su vez, pueden incrementar los niveles de WE, y por tanto, dado que este mecanismo funciona a modo de espiral de beneficios, conseguir una mejora tanto en el bienestar y la salud laboral como en los resultados organizacionales. A este respecto, otros autores identifican resultados positivos relacionados con más altos niveles de WE (desempeño extra-rol, empleabilidad, comportamiento innovador, satisfacción vital, compromiso con la organización, buena salud percibida, calidad del servicio y satisfacción del cliente, mejora de la productividad y resultados económicos y sociales) y resultados negativos relacionados con más bajos niveles de WE (absentismo, accidentes y daños, complicaciones psicosomáticas, tensión psicológica) (Schaufeli y Taris, 2014).

Luego, tomando como base la revisión de la literatura expuesta, es posible definir un modelo DRL rediseñado, con la inclusión de las prácticas de recursos humanos de alto rendimiento, STAR, como antecedentes. Dicho modelo se representa en la Figura 1, éste se seguirá en el análisis del ámbito de las organizaciones de Acción Social. 


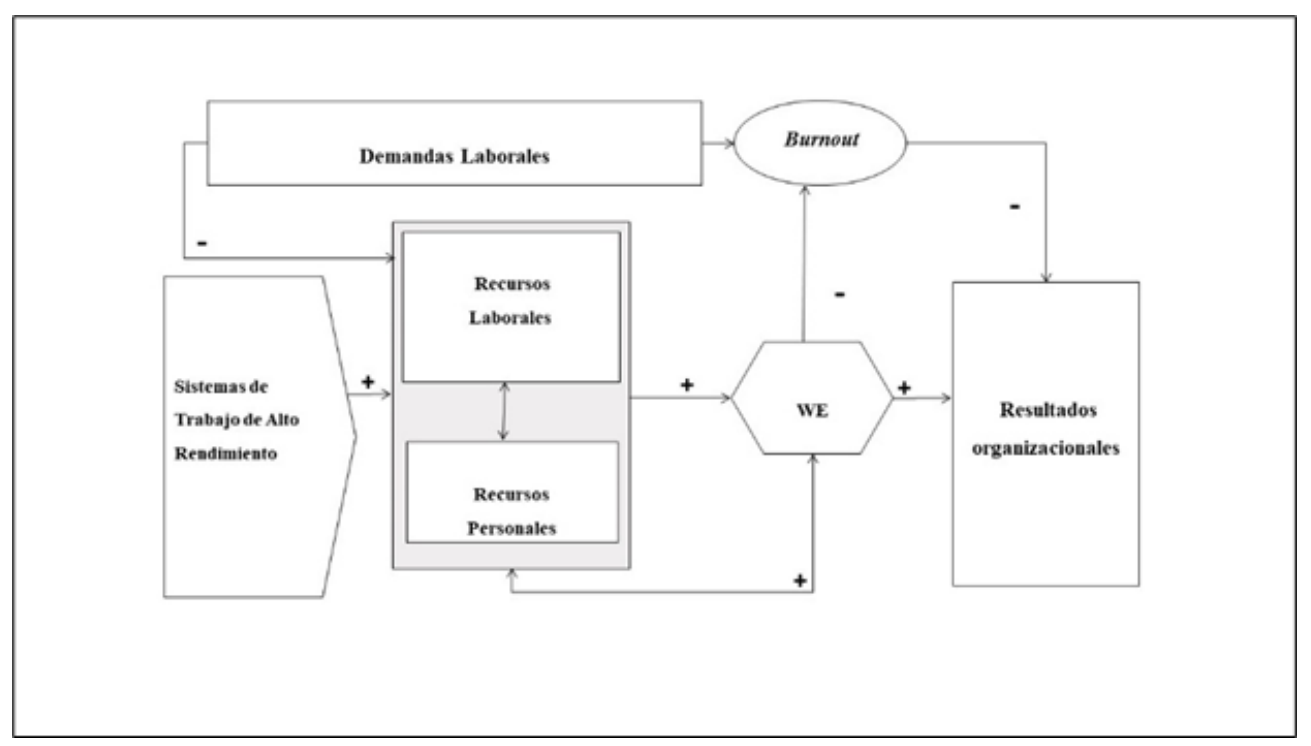

Figura. 1. Modelo DRL rediseñado

\section{Contexto de la investigación}

Atendiendo a lo establecido en la Ley 43/2015 del Tercer Sector de Acción Social "Las entidades del Tercer Sector de Acción Social son aquellas organizaciones de carácter privado, surgidas de la iniciativa ciudadana o social, bajo diferentes modalidades, que responden a criterios de solidaridad y de participación social, con fines de interés general y ausencia de ánimo de lucro, que impulsan el reconocimiento y el ejercicio de los derechos civiles, así como de los derechos económicos, sociales o culturales de las personas y grupos que sufren condiciones de vulnerabilidad o que se encuentran en riesgo de exclusión social" (BOE, 2015). Estas organizaciones surgieron, principalmente, para dar respuesta a necesidades de determinados colectivos vulnerables, apoyándose el sector público en su iniciativa para prestar servicios sociales y gestionar problemas de integración de estos grupos.

La política y los fondos europeos contribuyeron a que el TSAS se involucrase en las políticas de inclusión social y laboral. En lo relativo a su forma jurídica, se organizan principalmente como asociaciones, aproximadamente dos tercios del total, y en su evolución han ido conformándose en redes, a modo de federaciones y confederaciones por ámbitos de actuación (por ejemplo: discapacidad, infancia, etc.) (POAS, 2017). En el ámbito de la Acción Social en España, un 29,4 por ciento de las entidades se dedican a prestar servicios a personas con discapacidad (POAS, 2019). Este colectivo adquiere una relevancia muy especial actualmente, en la reciente revisión de los ODS (United Nations, 2020), éstos se han explicitado y conectado aún más con la necesidad de transformar el mundo para las personas con discapacidad, en particular, en un mundo cada vez más sostenible y centrado en las personas.

Dada la significancia del colectivo, y con el fin último de dar respuesta a la proposición de investigación y a los objetivos planteados, se seleccionó una organización perteneciente a Plena Inclusión España (integrada por 17 federaciones autonómicas, más Ceuta y Melilla, y que cuenta aproximadamente 900 asociaciones) (Plena Inclusión, 2020). Concretamente se seleccionó la Asociación Malagueña en favor de las Personas con Discapacidad Intelectual (Aspromanis), entidad privada, sin ánimo de lucro, creada en 1964 y declarada de utilidad pública en 1970, entidad del TSAS, cuya actividad económica se encamina a contribuir a la mejora de la calidad de vida de las personas con discapacidad intelectual y sus familias, luchando por sus derechos y prestando servicios de atención y apoyo en todos los ámbitos de su vida, llevando a cabo, para ello, actividades como terapia ocupacional, para la mejora de sus habilidades sociales y prácticas, formación y empleo, asesoramiento jurídico, apoyo a familias, servicio de ocio y tiempo libre. Aspromanis siempre ha considerado el capital humano de la organización como un factor estratégico, esencial para la consecución de su misión y elemento clave en la generación de valor y ventaja competitiva en la entidad (Aspromanis, 2020).

\section{Metodología}

Como metodología de investigación se siguió una combinación de métodos, tanto cuantitativos, como cualitativos (véase Tabla 1), que pasan a describirse a continuación. 
En primer lugar, con respecto a la identificación de las STAR puestas en marcha por la entidad, se realizaron dos entrevistas abiertas no estructuradas, al gerente y a la responsable del área de relaciones laborales y retribuciones de la organización. Se tomó como guía una combinación elaborada ad-hoc para la ocasión, basada en la encuesta de prácticas STAR (Lertxundi y Landeta, 2011; Stirpe y Revilla, 2013; Tang y Tang, 2012) y la metodología HERO (Acosta et al, 2011; Llorens, del Líbano y Salanova, 2009; Salanova et al, 2013) de organizaciones saludables.

Tabla. 1. Combinación de métodos de observación utilizados

\begin{tabular}{|l|l|l|}
\hline $\begin{array}{l}\text { Método de recopilación } \\
\text { de datos }\end{array}$ & Variable del marco teórico & Participantes \\
\hline $\begin{array}{l}\text { Entrevista } \\
\text { Encuesta de prácticas } \\
\text { STAR }\end{array}$ & Prácticas STAR & $\begin{array}{l}\text { Gerente } \\
\text { Responsable del área de } \\
\text { relaciones laborales y } \\
\text { retribuciones } \\
\text { Ocho directivos de nivel } \\
\text { intermedio }\end{array}$ \\
\hline $\begin{array}{l}\text { Cuestionario Maslach } \\
\text { Burnout Inventory- } \\
\text { General Survey (MBI-GS) }\end{array}$ & Burnout & 42 empleados \\
\hline Cuestionario UWES-9 & WE & Lempleados \\
\hline $\begin{array}{l}\text { Focus group y } \\
\text { observación directa }\end{array}$ & $\begin{array}{l}\text { Demandas laborales, recursos } \\
\text { laborales y personales, resultados } \\
\text { organizacionales }\end{array}$ & 35 empleados \\
\hline
\end{tabular}

Fuente: Elaboración propia.

Posteriormente, a través de entrevistas con ocho directivos de nivel intermedio, se analizó el grado de desarrollo de las prácticas STAR, para ello, se utilizó la encuesta de prácticas STAR, basándose en el modelo AMO (Ability, Motivation and Opportunity) (Appelbaum et al, 2000; Gardner et al, 2001; Guest, 1997). Esta encuesta estudia las dimensiones: habilidad, motivación y oportunidad; subdivididas en seis indicadores de prácticas organizacionales y de gestión de personas utilizadas en los procesos de selección, formación, retribución, trabajo en equipo, comunicación y participación. Estos indicadores, son medidos por 24 ítems (en una escala Likert del 1 al 7; $1=$ nunca se utilizan y $7=$ se utilizan siempre), de los cuales se procedió a eliminar el ítem referido a reparto de beneficios de la empresa, para su adaptación a Aspromanis, al no poder aplicarse por ser una entidad sin ánimo de lucro. Para responder a los ítems citados, siguiendo la metodología empleada por Lertxundi y Landeta (2011), se les pidió a los participantes encuestados, que pertenecían al nivel directivo de los distintos centros, que tomasen en consideración un horizonte temporal de tres años, y centrasen sus respuestas teniendo presentes a los profesionales de línea operativa (Véase Anexo).

En segundo lugar, se procedió a la medición de los niveles de burnout y WE presentes en la organización. Para la medición del burnout se utilizó el cuestionario Maslach Burnout Inventory-General Survey (MBIGS) de Maslach y Jackson (1986), adaptado por Bresó y otros (2007) en la Nota Técnica de Prevención (NTP) 732 del Instituto Nacional de Seguridad e Higiene en el Trabajo. Este instrumento en versión española consta de 15 ítems, que reflejan las tres dimensiones de burnout, agotamiento, cinismo y eficacia profesional. Cada una de las subescalas está constituida por 5, 4 y 6 ítems respectivamente, evaluados mediante una escala de respuesta tipo Likert $(0=\mathrm{Nunca} / 6=$ Siempre), siendo posible su comparación con una tabla de datos normativos facilitados en dicha nota técnica. En la medición del WE se utilizó la encuesta Utrecht Work Engagement Sale (UWES-9) (Schaufeli y Bakker, 2003). La escala se compone un total de 9 ítems que reflejan las tres dimensiones del WE: vigor, dedicación y absorción. Estos ítems son evaluados mediante una escala de respuesta tipo Likert (1=nunca/6=todos los días), siendo posible su comparación con una tabla de datos normativos para el contexto español. En la evaluación participó una muestra compuesta por 42 profesionales de Aspromanis (33,66 por ciento de un total de 125 profesionales que componían su plantilla total en el momento de la investigación). Participaron profesionales de diversos ámbitos ocupacionales de la organización, tanto de atención directa (cuidadores, encargados y monitores de taller), como personal de apoyo (administrativos y servicio doméstico).

En tercer lugar, se implementó un método, etnográfico (Hammersley, 2007), incluyendo observación directa y focus group, dirigidos a los principales stakeholders de la entidad (empleados, directivos). Es preciso hacer notar que uno de los investigadores ha trabajado en entidades del TSAS durante 26 años, en el ámbito de la gestión de personas, lo que aporta un conocimiento diferenciado, y de alto valor añadido, para 
llevar a cabo el análisis de los resultados, en su contexto. Concretamente se realizó un taller formativo sobre el modelo DRL y el WE, y en el desarrollo del mismo se procedió a aplicar la técnica del grupo focal (focus group), como entrevista grupal, informal, diseñada para producir datos de manera más accesible gracias al uso explícito de la interacción de sus participantes (Morgan, 1997; García y Mateo, 2000), llevándose a cabo en un ambiente más permisivo y no directivo (Krueger, 1991). Hay que tener en cuenta que el focus group es una técnica que permite generar una aproximación novedosa a una temática de investigación, a través del consenso al que llegan los participantes en la experiencia.

En el desarrollo del taller se impartió una formación sobre el modelo DRL y el concepto de WE, las dimensiones que lo componen y sus efectos en la organización. En la segunda parte del mismo se invitó a los participantes, 35 empleados de Aspromanis a reflexionar sobre las demandas laborales de su trabajo, los recursos laborales y personales, así como sobre sus percepciones sobre los resultados organizaciones, tanto positivos como negativos, que asociaban al WE. Además, reflexionaron sobre su percepción respecto a las prácticas STAR como antecedentes del WE.

\section{Resultados}

En relación con las prácticas STAR llevadas a cabo por la organización, éstas se centran en diversas áreas de acción: comunicación, selección, retribución, participación, formación, conciliación de vida familiar y laboral, estabilidad laboral, etc., mediante programas específicos que las desarrollan. En la Tabla 2 se recogen las prácticas STAR identificadas en la entidad, a partir de las entrevistas llevadas a cabo con los informantes estratégicos. Además, atendiendo a las percepciones de los directivos participantes, se valoró su grado de utilización, a partir de los resultados de la encuesta STAR. En este sentido, en cuanto a la media global, se obtuvo un resultado de 5,50 puntos, lo que indica que los encuestados opinan que, de forma global, la utilización de las prácticas STAR se sitúa en un grado de incidencia alta. En esta encuesta destaca entre estas prácticas la comunicación como la mejor situada, seguida de la selección de personal, el trabajo en equipo y la participación de los empleados.

Tabla. 2. Sistemas de Trabajo de Alto Rendimiento desarrollados en Aspromanis

\begin{tabular}{|c|c|}
\hline Área de acción & Acciones \\
\hline \multirow[t]{4}{*}{ Selección } & $\begin{array}{l}\text { Centrada en la adecuación de la titulación al puesto a ocupar. Personal acreditado } \\
\text { profesionalmente. }\end{array}$ \\
\hline & $\begin{array}{l}\text { Realizada mediante equipo multidisciplinar (assessment center) en puestos clave de la } \\
\text { organización. }\end{array}$ \\
\hline & Ofertas de promoción interna de los empleados. \\
\hline & $\begin{array}{l}\text { Procesos de acogida de nuevos empleados: tutorización por otro empleado o equipo de } \\
\text { trabajo. }\end{array}$ \\
\hline \multirow[t]{3}{*}{ Formación } & Planes de formación: específica y polivalente. \\
\hline & Planes de cualificación profesional. \\
\hline & Iniciación a la gestión del conocimiento. \\
\hline Desempeño & Evaluaciones según puesto de trabajo. \\
\hline $\begin{array}{l}\text { Políticas } \\
\text { retributivas }\end{array}$ & $\begin{array}{l}\text { Aplicación del convenio colectivo del sector. } \\
\text { Acuerdo inter-partes: abono de festivos trabajados en mayor cuantía que la establecida } \\
\text { en el convenio colectivo. }\end{array}$ \\
\hline \multirow[t]{2}{*}{ Participación } & Círculos de calidad. \\
\hline & Reuniones de evaluación del servicio, buzón de sugerencias y banco de ideas. \\
\hline \multirow[t]{6}{*}{ Comunicación } & Difusión del plan estratégico. \\
\hline & Difusión cuentas anuales publicadas en web. \\
\hline & Difusión del boletín de noticias a toda la plantilla. \\
\hline & Página web corporativa con difusión de la misión, visión y valores. \\
\hline & Difusión del código ético, en centros de trabajo y página web. \\
\hline & Difusión información relevante en tablones de anuncios. \\
\hline \multirow[t]{2}{*}{ Engagement } & Autonomía en la toma de decisiones de los empleados. \\
\hline & Equipos de trabajo de alto rendimiento. \\
\hline Conciliación de & Especial protección de los riesgos laborales durante el embarazo: adaptación del puesto \\
\hline
\end{tabular}




\begin{tabular}{|l|l|}
\hline Área de acción & Acciones \\
\hline \multirow{4}{*}{ vida familiar } & y suspensión del contrato en caso de no ser viable la adaptación. \\
\cline { 2 - 2 } & Reducciones de jornada por cuidado de hijos y familiares. \\
\cline { 2 - 2 } & Excedencias por cuidado de hijos y familiares. \\
\cline { 2 - 2 } & Medidas de flexibilización horaria por solicitud de los trabajadores. \\
\hline $\begin{array}{l}\text { Estabilidad } \\
\text { laboral }\end{array}$ & Alto índice de empleo estable, contratación indefinida del 85 por ciento de la plantilla. \\
\hline $\begin{array}{l}\text { Relaciones } \\
\text { interpersonales }\end{array}$ & $\begin{array}{l}\text { Fomento del buen clima laboral y relaciones interpersonales mediante reuniones de } \\
\text { cohesión de grupos, debate y dinámicas de grupo que incluyen métodos de narración } \\
\text { de experiencias. }\end{array}$ \\
\hline
\end{tabular}

Fuente: Elaboración propia.

Estos resultados indican que la entidad centra sus esfuerzos en que los profesionales se mantengan informados de lo concerniente a su trabajo, se preocupa por una adecuada selección del personal, potencia la participación de los empleados y está muy presente el trabajo en equipo. Finalmente, la formación y la retribución se sitúan en valores de incidencia media.

En la medición del burnout y del WE participaron 42 profesionales de Acción Social, de la organización estudiada, de los cuales el 33 por ciento eran hombres y el 67 por ciento mujeres, aproximadamente un 50 por ciento contaban con estudios universitarios, y la media de edad era de 41 años, con 12 años de promedio trabajando en el ámbito de la organización. La fiabilidad de las escalas arroja una consistencia interna razonable, dado que en todos los casos los coeficientes superan el valor recomendado de 0,60 , para el caso del WE como dimensión única, y en los casos del burnout, con sus tres dimensiones según la literatura (Nunnaly y Bernstein, 1994). En la Tabla 3 se muestran las medias, las desviaciones estándar y los coeficientes de fiabilidad. En los cálculos se ha utilizado el software IBM SPSS Statistics v26.

Tabla. 3. Media, desviación típica y correlaciones de las escalas de burnout y WE

\begin{tabular}{|l|l|l|l|}
\hline & Media & $\begin{array}{l}\text { Desviación } \\
\text { típica }\end{array}$ & $\begin{array}{l}\boldsymbol{\alpha} \text { Cronbach (elementos } \\
\text { estandarizados) }\end{array}$ \\
\hline WE & 5,31 & 0,4 & 0,664 \\
\hline Agotamiento & 1,43 & 1,6 & 0,891 \\
\hline Cinismo & 0,5 & 0,73 & 0,828 \\
\hline $\begin{array}{l}\text { Eficiencia } \\
\text { profesional }\end{array}$ & 4,92 & 0,92 & 0,882 \\
\hline
\end{tabular}

Fuente: Elaboración propia.

Comparando los resultados con el baremo normativo de burnout adaptado por Bresó y otros (2007), los profesionales de este estudio muestran, de acuerdo con los puntos de corte establecidos, niveles de agotamiento $(1,43)$ y de cinismo $(0,50)$ medio bajo, y un nivel de eficacia profesional $(4,92)$ medio alto, datos consistentes con niveles bajos de burnout.

En cuanto a la medición por promedio realizada en la entidad en relación con el WE, los resultados del WE existente de manera global $(5,31)$, reflejan niveles altos en la muestra estudiada (Schaufeli y Bakker, 2003; Salanova y Schaufeli, 2009).

En el modelo DRL rediseñado se ha definido una relación de antítesis entre los constructos WE y el burnout, la medición de ambos constructos nos permite poder realizar un análisis de correlaciones entre las dimensiones agotamiento, cinismo y eficiencia profesional y el WE, obteniéndose en este sentido correlaciones negativas, significativas, en la muestra estudiada, entre el WE y el ítem cinismo (Tabla 4). 
Tabla. 4. Correlaciones entre WE y las dimensiones del burnout

\begin{tabular}{|l|l|l|l|l|}
\hline & WE & Agotamiento & Cinismo & $\begin{array}{l}\text { Eficiencia } \\
\text { profesional }\end{array}$ \\
\hline WE & 1 & & & \\
\hline Agotamiento &,- 251 & 1 & & \\
\hline Cinismo & $-0,403^{* *}$ &, $643^{* *}$ & 1 & \\
\hline Eficiencia profesional & 0,118 & 0,048 & 0,043 & 1 \\
\hline
\end{tabular}

Correlación de Pearson **. La correlación es significativa en el nivel 0,01.

Fuente: Elaboración propia.

En lo referente a los resultados del focus group, se animó a los participantes a encontrar por ellos mismos los elementos de demandas laborales, recursos laborales y personales, resultados positivos y negativos en la organización, que integran el modelo DRL, presentes en su día a día y su actividad laboral. Este tema a debatir no era conocido por los participantes hasta el momento de iniciarse el debate, por lo que fue necesario hacer una presentación del tema y los conceptos relacionados, a modo de formación previa, para proporcionarles herramientas con las que pudieran generar un análisis que aportase fluidez al taller. Posteriormente, se les instó a debatir sobre qué demandas laborales percibían de manera más frecuente en relación con su trabajo, qué recursos laborales y personales estaban a su vez presentes para hacer frente a estas demandas, si consideraban que las prácticas organizacionales estaban siendo utilizadas por la organización y si consideraban que éstas podrían ser responsables de generar más recursos laborales y personales con los que hacer frente a las demandas laborales. Para la toma de datos se procedió utilizando el cuadro de clasificación de demandas y recursos (Schaufeli y Taris, 2014), realizándose una adaptación de este método a la mencionada técnica del grupo focal, mediante un debate abierto, para la identificación de las principales demandas laborales y recursos laborales y personales, resultados positivos y negativos que los participantes percibían más presente en la organización. El debate suscitado en el focus group dio como resultado una identificación de demandas y recursos laborales, y recursos personales, así como de los resultados positivos y negativos, identificados por los profesionales en la entidad de Acción Social, que quedan reflejados en la Tabla 5 .

Tabla. 5. Identificación de Demandas, Recursos y Resultados

\begin{tabular}{|c|c|}
\hline \multicolumn{2}{|l|}{ Demandas Laborales } \\
\hline \multicolumn{2}{|c|}{$\begin{array}{l}\text { Por contactos con usuarios o clientes, sobrecarga cualitativa de trabajo, riesgos laborales, presión de la tarea, } \\
\text { planificación. }\end{array}$} \\
\hline Recursos Laborales & Recursos Personales \\
\hline $\begin{array}{l}\text { Desarrollo profesional, clima de innovación, reto laboral, } \\
\text { conocimiento, liderazgo y confianza en la gestión, participación en la } \\
\text { toma de decisiones, orgullo profesional, apoyo social de compañeros y } \\
\text { supervisor, cohesión/armonía de equipo. }\end{array}$ & $\begin{array}{l}\text { CapPsic (optimismo, resiliencia, autoeficacia, } \\
\text { esperanza), motivación intrínseca, creatividad, } \\
\text { innovación, autonomía, pertenencia, } \\
\text { competencia. }\end{array}$ \\
\hline Resultados organizativos (positivos) & Resultados organizativos (negativos) \\
\hline $\begin{array}{l}\text { Empleados: Satisfacción vital, compromiso con la organización, } \\
\text { empleabilidad, buena salud percibida, comportamiento innovador. } \\
\text { Clientes: calidad del servicio y satisfacción del cliente. } \\
\text { Organización: mejora de la productividad y resultados económicos y } \\
\text { sociales. }\end{array}$ & $\begin{array}{l}\text { Absentismo, accidentes y daños, intención de } \\
\text { abandono, mala salud física, complicaciones } \\
\text { psicosomáticas, tensión psicológica. }\end{array}$ \\
\hline
\end{tabular}

Fuente: Elaboración propia.

\section{Discusión}

El presente trabajo atendiendo a los objetivos definidos previamente, en primer lugar, propone un rediseño del modelo DRL, incorporando las prácticas STAR, a partir de la revisión de la literatura. Posteriormente, dada la combinación de métodos de recopilación de información directa en Aspromanis, tanto cuantitativos como cualitativos, se ha podido cumplir por un lado con el objetivo de medición de los niveles de burnout y el WE de los profesionales de la Acción Social, en esta entidad. En cuanto a los resultados de la medición de los niveles del WE, se obtiene que, de forma global, se dan niveles altos, mientras que en lo relativo al burnout, los niveles son medio-bajos (Figura 2). Y además se han analizado las correlaciones entre ambos constructos, identificándolos como polos opuestos, en particular, a través de las correlaciones significativas 
negativas entre WE y cinismo. Por último, en relación con el objetivo referente a conocer las percepciones de los profesionales de las entidades del TSAS sobre las demandas y recursos laborales, así como de los recursos personales, y su relación con las STAR y los resultados de la organización, respecto a las demandas laborales, que subyacen tras el burnout, los profesionales, manifestaron que los contactos con usuarios o clientes, suponían una de las más difíciles de afrontar, debido a que la atención directa hacia personas con discapacidad conlleva a veces procesos casi individualizados, en cuanto al servicio ofrecido, y existen necesidades de atención muy diversas a las que hacer frente. Asimismo, las demandas del desempeño, la sobrecarga cualitativa, los problemas de planificación y la presión de la tarea, forman parte de las demandas más relacionadas con los factores organizativos que influyen en un mejor desempeño de la tarea. Los riesgos laborales constituyen otra de las demandas que generan preocupación en los profesionales de la entidad, aunque éstos identifican la existencia de un entorno laboral seguro, consideran según sus opiniones que es un aspecto que debe ser siempre tenido en cuenta. Para amortiguar estas demandas son clave, en opinión de los profesionales, los recursos laborales, considerando como los más destacables, los siguientes, el desarrollo profesional, el clima de innovación, la confianza en la gestión, la participación en las decisiones, el orgullo profesional, el apoyo social y la armonía de equipo.

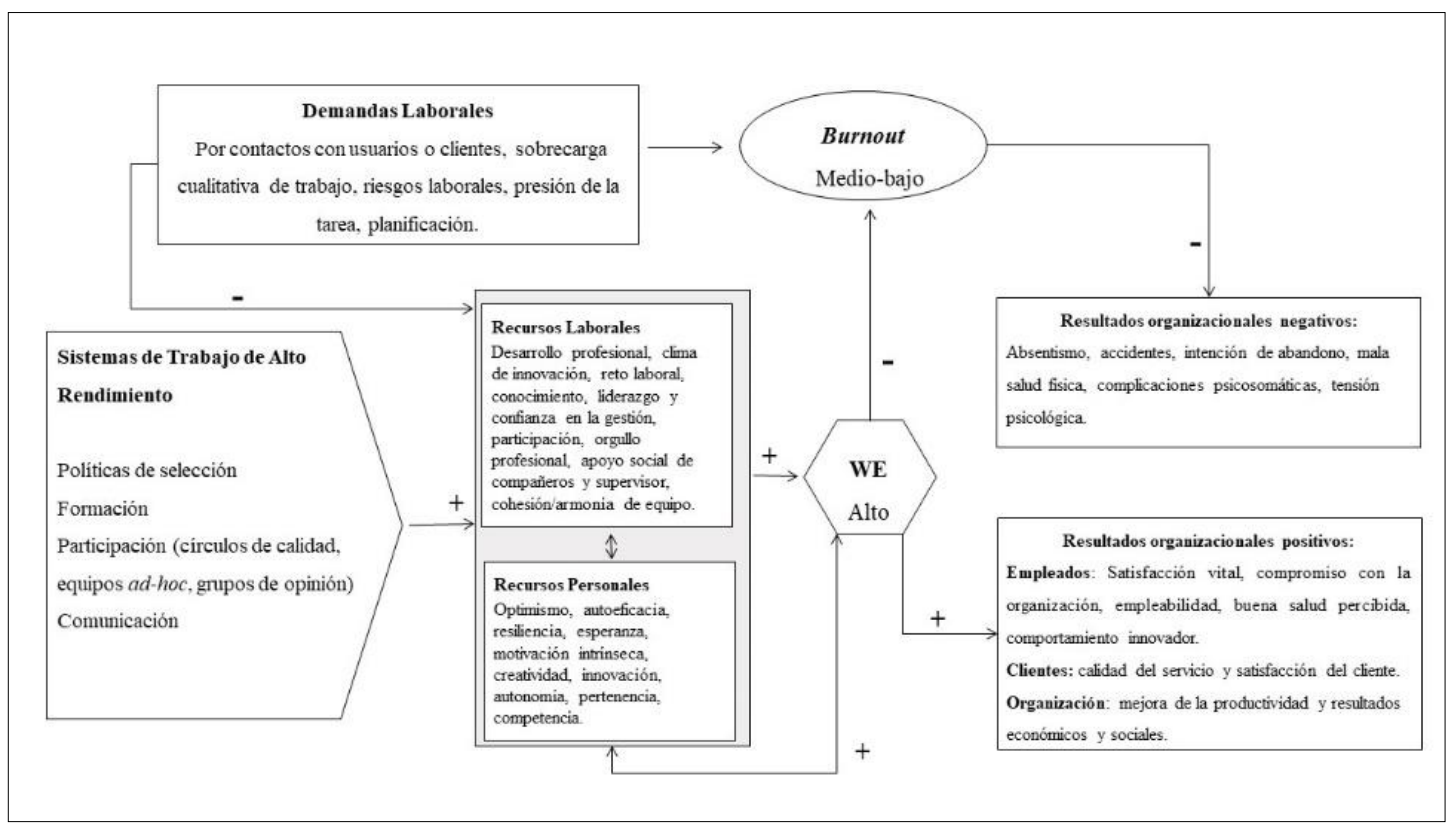

Figura. 2. Modelo de DRL rediseñado en el caso del TSAS

Asimismo, los participantes manifestaron que en su opinión, los recursos laborales influían en los recursos personales, tales como el optimismo, la resiliencia, la autoeficacia y la esperanza, y que otros recursos personales, como la creatividad o la innovación, son de especial interés en su trabajo, dado que el ser creativos e innovadores permite llevar a cabo cambios en la planificación de actividades a realizar por las personas usuarias del servicio, convirtiéndolas en más atractivas y motivadoras para ellas, e incluso para los propios profesionales. Identificaron a su vez, otros recursos personales, como autonomía, sentido de pertenencia y sensación de competencia.

En relación con las prácticas STAR los profesionales del sector identifican que su implementación puede ayudar a la generación de recursos laborales y personales, en particular las relacionadas con la selección de profesionales, la formación y participación de los empleados (círculos de calidad, equipos ad-hoc para cuestiones específicas y grupos de opinión). Estas prácticas coinciden con las de mayor incidencia puestas en marcha por la organización analizada, a excepción, de la de comunicación, no identificada por los empleados como prioritaria respecto de la generación de recursos, pero relevante desde el punto de vista de gestión empresarial, para que los empleados cuenten con toda la información necesaria para el desempeño de su actividad. Por su parte, la formación es un aspecto clave para los empleados, por su impacto en los recursos laborales y personales, y es un aspecto a potenciar por parte de la organización estudiada.

En cuanto a los resultados organizacionales positivos debatidos en el focus group destacaron la existencia de tres ámbitos (empleados, clientes y organización) en los que los recursos laborales y personales, como generadores de WE, ejercen una clara influencia. En cuanto a los empleados, encontraron, según sus percepciones, que los resultados podrían identificarse como: satisfacción vital, compromiso con la organización, empleabilidad, buena salud percibida, comportamiento innovador. 
Respecto a los clientes destacaba la calidad en el servicio y la satisfacción como un resultado incluso posible de medir. Y finalmente en cuanto a la organización apreciaban una mejora de la productividad y resultados económicos. Asimismo, se les pidió que identificaran resultados negativos que podrían manifestarse a partir de la falta prácticas de recursos humanos de alto rendimiento y de WE, argumentando los participantes que su escasa presencia podría conllevar mayores índices de absentismo, accidentes laborales, tensión psicológica y mala salud física y psíquica de los empleados.

\section{Conclusiones}

Como conclusión general de esta investigación se desprende que atendiendo a las percepciones de los profesionales es posible potenciar el WE de los empleados en las entidades del TSAS a través de la implementación de prácticas STAR, para lograr mejorar la calidad del servicio y mejores resultados organizacionales y sociales.

Este trabajo de investigación, desde la perspectiva de las organizaciones de Acción Social, permite evidenciar, de un modo descriptivo, cómo el WE de los empleados, con niveles altos, y el burnout, con niveles medio-bajos, se reflejan como fenómenos opuestos. Los empleados de las organizaciones del TSAS experimentan demandas laborales, subyacentes en el síndrome de estar quemado, propias de su tarea y del tipo de colectivo que atienden. En este sentido, es prioritario conocer las demandas de los empleados de la organización, en relación con el binomio tarea-usuario. Los efectos negativos de estas demandas pueden verse modulados por los recursos laborales y personales, en este sentido las prácticas STAR llevadas a cabo en la organización pueden influir positivamente en la generación de dichos recursos o en su potenciación, concretamente las relacionadas con la selección, la formación y la participación, se refieren por los profesionales como las más destacables del modelo DRL rediseñado, en el caso de entidades del TSAS. Estos recursos, tanto laborales como personales, potencialmente impactan positivamente en el WE de los empleados, en opinión de los profesionales, y éste a su vez influye positivamente en los resultados organizacionales, que concretamente en el caso de este sector pueden clasificarse, en positivos, buena salud percibida de los empleados, comportamiento innovador, calidad del servicio, mejora de la productividad, entre otros, en el caso de ser alto, o en negativos, absentismo, deterioro de la salud, alta rotación, entre otros, en caso de niveles altos de burnout, y por tanto bajos de WE.

Luego los departamentos de Gestión de Personas de las entidades del TSAS deben diseñar prácticas STAR con el objetivo de modular las demandas laborales, antecedentes del burnout, y que potencien los recursos tanto laborales como personales de los profesionales del sector para incrementar su nivel de WE. La literatura al respecto y, en opinión de los profesionales participantes en esta investigación, se logran resultados positivos, entre otros, incrementos en la calidad del servicio y bienestar de los usuarios, y por tanto, dado el impacto social de las entidades del TSAS, estos resultados serán sin duda también sociales, una sociedad más inclusiva y sostenible.

Como limitaciones, en primer lugar, habría que profundizar más en los estudios en este sector, ya que son bastante escasos, y los existentes han sido realizados en corte transversal, por lo que futuros estudios longitudinales, podrían aportar mayores datos comparativos sobre el WE, pudiendo abordarse incluso a nivel metodológico continuo, junto con otras encuestas de satisfacción laboral, burnout, recuperación tras la jornada, etc. Otra limitación se puede encontrar en que se analiza una única organización, hay que ser conscientes de la dificultad que supone obtener información directa, sobre las percepciones de los empleados, y los directivos, sobre temas tan sensibles. La muestra obtenida, limitada, para los análisis cuantitativos, no ha permitido discriminar resultados y correlaciones para las distintas subescalas del WE, sería preciso ampliar la muestra de empleados de la propia entidad o de otras entidades del TSAS para generalizar los resultados. Por otra parte, en relación con la información cualitativa obtenida, también existen dificultades de extrapolación a otras organizaciones que atiendan a otros colectivos, pero este trabajo supone un marco de aproximación para otras investigaciones.

Finalmente, como futuras líneas de investigación podría llevarse a cabo la aplicación del modelo a una muestra más amplia de entidades del TSAS, donde se pudiese validar el modelo de relaciones de los constructos a través de ecuaciones estructurales, lo cual se plantea por parte de los investigadores en este momento.

\section{Referencias bibliográficas}

Acosta, H., Salanova, M., Llorens, S. (2011) ¿Qué prácticas organizacionales saludables son más frecuentes en las empresas? Un análisis cualitativo. Recuperado en: http://repositori.uji.es/xmlui/bitstream/handle/10234/77366/fr_2011_9_1.pdf?sequence=1\&isAllowed=y. 
Appelbaum, E., Bailey, T., Berg, P. B., Kalleberg, A. L., Bailey, T. A. (2000) Manufacturing advantage: Why highperformance work systems pay off. Champaign, IL: Employment Policy Research Network.

Arocas, R. L., Torres, J. C. (2006) Las prácticas de alto rendimiento en recursos humanos: el caso español. Tec Empresarial, $\quad \mathrm{N}^{\mathrm{o}} \quad 1, \mathrm{vol}$, $1, \quad$ pp. 26-30. Recuperado en: https://revistas.tec.ac.cr/index.php/tec empresarial/article/view/874.

Aspromanis (2020) Aspromanis. Recuperado en: http://aspromanis.org/.

Bakker, A. B., Demerouti, E. (2007) The job demands-resources model: State of the art. Journal of Managerial Psychology, No 22, vol. 3, pp. 309-328. DOI: 10.1108/02683940710733115.

Bakker, A. B., Demerouti, E. (2008) Towards a model of work engagement. Career Development International, $\mathrm{N}^{\mathrm{o}} 13$, vol. 3, pp. 209-223. DOI: 10.1108/13620430810870476.

Barrick, M. R., Thurgood, G. R., Smith, T. A., Courtright, S. H. (2015) Collective organizational engagement: Linking motivational antecedents, strategic implementation, and firm performance. Academy of Management Journal, $\mathrm{N}^{\mathrm{o}}$ 58, vol. 1, pp. 111-135. DOI: 10.5465/amj.2013.0227.

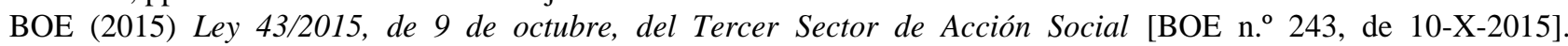
Recuperado en https://www.boe.es/buscar/act.php?id=BOE-A-2015-10922.

Bresó, E., Salanova, M., Schaufelli, W., Nogareda, C. (2007) NTP 732: Síndrome de estar quemado por el trabajo burnout (III): instrumento de medición. Instituto Nacional de Seguridad e Higiene en el Trabajo. Recuperado en: https://www.insst.es/documents/94886/327446/ntp_732.pdf/bf45e644-2986-42b0-b9a5-ce5bef2917bd.

Comos, C (2018) El Tercer Sector de Acción Social y su vinculación a la economía social. Revista Española del Tercer Sector, $\mathrm{N}^{\circ} 38$, pp. 193-196.

Demerouti, E., Bakker, A. B., Nachreiner, F., Schaufeli, W. B. (2001) The job demands-resources model of burnout. Journal of Applied Psychology, No 86, vol. 3, pp. 499. DOI: 10.1037/0021-9010.86.3.499.

Durán, M. A., Manteca, A. J. (2013) Engagement en profesionales de la acción social. Diferencias entre Administración y Tercer Sector desde la perspectiva del modelo de demandas y recursos laborales. Documentos de Trabajo Social, No 51, pp. 45-68.

Durán, M. A., Rey, L., Extremera, N. (2004) Análisis del contexto laboral y del perfil sociodemográfico de una muestra de profesionales que atienden a personas con discapacidad intelectual. Siglo Cero: Revista Española sobre Discapacidad Intelectual, $\mathrm{N}^{\mathrm{0}} 35$, vol. 212, pp. 18-32.

European Commission (2016) Europe 2020: A strategy for smart, sustainable and inclusive growth: Communication from the commission. Publications Office of the European Union. Recuperado en: http://eurlex.europa.eu/LexUriServ/LexUriServ.do?uri=COM:2010:2020:FIN:EN:PDF.

Extremera, N., Durán, M. A., Rey, L. (2005) La inteligencia emocional percibida y su influencia sobre la satisfacción vital, la felicidad subjetiva y el engagement en trabajadores de centros para personas con discapacidad intelectual. Ansiedad y estrés, $\mathrm{N}^{\mathrm{o}} 11$, vol. 1, pp. 63-73.

Fidalgo, M. (2006a) NTP 704: Síndrome de estar quemado por el trabajo o burnout (I): definición y proceso de generación. Madrid: Instituto Nacional de Seguridad e Higiene en el Trabajo. Recuperado en: https://www.insst.es/documents/94886/327446/ntp_704.pdf/9a205bee-9bd7-4221-a1ae-39b737974768.

Fidalgo, M. (2006b) NTP 705: Síndrome de estar quemado por el trabajo o burnout (II): consecuencias, evaluación y prevención. Madrid: Instituto Nacional de Seguridad e Higiene en el Trabajo. Recuperado en: https://www.insst.es/documents/94886/327446/ntp_705.pdf/a6901ca1-e0a3-444d-96dd-419079da204d.

García, M. M., Mateo, I. (2000) El grupo focal como técnica de investigación cualitativa en salud. Atención primaria, $\mathrm{N}^{\mathrm{o}} 25$, vol. 3, pp. 181-186.

Hammersley, M.A.P (2007) Ethnography: Principles in Practice. Routledge.

Kahn, W. A. (1990) Psychological conditions of personal engagement and disengagement at work. Academy of Management Journal, $\mathrm{N}^{\mathrm{o}} 33$, vol. 4, pp. 692-724. DOI: 10.5465/256287.

Krueger, R. A. (1991) El grupo de discusión: guía práctica para la investigación aplicada. Madrid: Pirámide.

Lertxundi, A., Landeta, J. (2011) The moderating effect of cultural context in the relation between HPWS and performance: An exploratory study in Spanish multinational companies. The International Journal of Human Resource Management, № 22, vol. 18, pp. 3949-3967. DOI: 10.1080/09585192.2011.610942.

Lertxundi, A., Landeta, J. (2012) The dilemma facing multinational enterprises: Transfer or adaptation of their human resource management systems. The International Journal of Human Resource Management, $\mathrm{N}^{\circ}$ 23, vol. 9, pp. 17881807. DOI: 10.1080/09585192.2011.610940.

Llorens, S., del Líbano, M., Salanova, M. (2009) Modelos teóricos de salud ocupacional. Psicología de la Salud Ocupacional. Madrid: Síntesis, pp. 63-93.

Llorens, S., Salanova, M., Torrente, P., Acosta, H. (2013) Interventions to Promote Healthy Resilient Organizations (HERO) from positive psychology. Salutogenic organizations and change: The concepts behind organizational health intervention research. Dordrecht: Springer, pp. 91-106.

Luthans, F., Avolio, B. J., Avey, J., Norman, S. (2007) Positive psychological capital: Measurement and relationship with performance and satisfaction. Personnel Psychology, $\mathrm{N}^{\mathrm{o}}$ 60, pp. 541-572. DOI: 10.1111/j.17446570.2007.00083.x.

Luthans, F., Luthans, K. W., Luthans, B. C. (2004) Positive psychological capital: Beyond human and social capital. Business Horizons, No 47, vol. 1, pp. 45-50. DOI: 10.1016/j.bushor.2003.11.007. 
Maslach, C., Jackson, S. E. (1981) The measurement of experienced burnout. Journal of Occupational Behavior, № 2 , vol. 2, pp. 99-113. DOI: 10.1002/job.4030020205.

Maslach, C., Jackson, S.E. (1986) MBI: Maslach Burnout Inventory; manual research edition. Palo Alto, CA: University of California.

Maslach, C., Leiter, M. P. (2001) Burnout and health. Handbook of health psychology. Mahwah, NJ: Lawrence Erlbaum Associates, pp. 415-426.

Maslach, C., Schaufeli, W. B., Leiter, M. P. (2001) Job burnout. Annual Review of Psychology, № 52, vol. 1, pp. 397422. DOI: 10.1146/annurev.psych.52.1.397.

Morgan, D. L. (1997) Focus groups as qualitative Research. Sage.

Nunnaly, J.C., Bernstein, I.H. (1994) Psychometric Theory. McGraw-Hill.

Peiró, J. M. (2004) El sistema de trabajo y sus implicaciones para la prevención de riesgos psicosociales en el trabajo. Universitas psychologica, $\mathrm{N}^{\mathrm{o}}$ 3, vol. 2, pp. 179-186.

Park, S., Kim, J., Park, J., Lim, D. H. (2018) Work engagement in nonprofit organizations: A conceptual model. Human Resource Development Review, $\mathrm{N}^{\mathrm{o}} 17$, vol. 1, pp. 5-33.

Plena Inclusión (2020) Plena Inclusión. Recuperado en: https://www.plenainclusion.org/.

POAS (2015) El tercer sector de acción social en 2015. Impacto de la crisis. Recuperado en: http://www.plataformaong.org/.

POAS (2017) Nuestro COMPROMISO con la sociedad. III Plan Estratégico del Tercer Sector de Acción Social $2017-$ 2021. Recuperado en: http://www.plataformatercersector.es/.

POAS (2019) El tercer sector de acción social en España 2019. Nuevos horizontes para un nuevo contexto sociopolítico. Recuperado en: http://www.plataformaong.org/.

Salanova, M., Llorens, S. (2008) Estado actual y retos futuros en el estudio del burnout. Papeles del Psicólogo: Revista del Colegio Oficial de Psicólogos, № 29, vol. 1, pp. 59-67.

Salanova, M., Llorens, S., Acosta, H., Torrente, P. (2013) Positive interventions in organizations. Terapia psicológica, No 31, vol. 1, pp. 101-113. Recuperado en: https://www.redalyc.org/pdf/785/78559051009.pdf.

Salanova, M., Martínez, I. M., Llorens, S. (2005) Psicología organizacional positiva. Psicología de la Organización. Madrid: Pearson Prentice Hall, pp. 349-376.

Salanova, M., Schaufeli, W. (2009) El engagement en el trabajo. Cuando el trabajo se convierte en pasión. Madrid: Alianza Editorial.

Salanova, M., Schaufeli, W. B., Llorens, S., Peiró, J. M., Grau, R. (2000) Desde el 'burnout' al 'engagement': ¿una nueva perspectiva? Journal of Work and Organizational Psychology, № 16, vol. 2, pp. 117-134.

Schaufeli, W. B., Bakker, A. B. (2003) Utrecht work engagement scale: Preliminary manual. Occupational Health Psychology Unit. Utrecht: Utrecht University, Vol. 26.

Schaufeli, W.B., Enzmann, D. (1998) The burnout companion to study and practice: a critical analysis. London: Taylor Francis.

Schaufeli, W. B., Salanova, M., González-Romá, V., Bakker, A. B. (2002) The measurement of engagement and burnout: A two sample confirmatory factor analytic approach. Journal of Happiness Studies, No 3, vol. 1, pp. 71-92. DOI: $10.1023 / \mathrm{A}: 1015630930326$.

Schaufeli, W. B., Taris, T. W. (2014) A critical review of the job demands-resources model: Implications for improving work and health. Bridging occupational, organizational and public health. Dordrecht: Springer, pp. 43-68.

Seligman, M., Csikszentmihalyi, M. (2014) Positive psychology: An introduction. Flow and the foundations of positive psychology. Dordrecht: Springer, pp. 279-298.

Stirpe, L., Revilla, A. J. (2013) Efectos de la contratación temporal sobre los resultados de los sistemas de trabajo de alto rendimiento. Universia Business Review, $\mathrm{N}^{\mathrm{o}} 39$, pp. 14-31. Recuperado en: https://journals.ucjc.edu/ubr/article/view/884.

Tang, T. W., Tang, Y. Y. (2012) Promoting service-oriented organizational citizenship behaviors in hotels: the role of high-performance human resource practices and organizational social climates. International Journal of Hospitality Management, № 31, vol. 3, pp. 885-895. DOI: 10.1016/j.ijhm.2011.10.007.

Thompson, K. R., Lemmon, G., Walter, T. J. (2015) Employee Engagement and Positive Psychological Capital. Organizational Dynamics, No 44, vol. 3, pp. 185-195. DOI: 10.1016/j.orgdyn.2015.05.004.

United Nations (2020) \#Envision2030: 17 goals to transform the world for persons with disabilities. Recuperado en: https://www.un.org/development/desa/disabilities/.

Van de Voorde, F. C., Veld, M., Van Veldhoven, M. J. P. M. (2016) Connecting empowerment-focused HRM and labor productivity to work engagement: The mediating role of job demands and resources. Human Resource Management Journal, No 26, vol. 2, pp. 192-210. DOI: 10.1111/1748-8583.12099. 


\section{ANEXO: Encuesta de prácticas STAR}

Según su estimación, valore la utilización de los sistemas de trabajo sobre Recursos Humanos en su organización. Puntúe cada ítem en una escala del 1 al 7.

\begin{tabular}{|l|l|l|l|l|l|l|}
\hline 1 & 2 & 3 & 4 & 5 & 6 & 7 \\
\hline Nunca & $\begin{array}{l}\text { De vez en } \\
\text { cuando }\end{array}$ & A veces & Normalmente & $\begin{array}{l}\text { Muy a } \\
\text { menudo }\end{array}$ & $\begin{array}{l}\text { Muchísimas } \\
\text { veces }\end{array}$ & Siempre \\
\hline
\end{tabular}

\begin{tabular}{|c|c|}
\hline A los procesos de selección de personal se les otorga mucha importancia & \\
\hline $\begin{array}{l}\text { En la selección, las referencias personales de tipo "lazo familiar" son tan } \\
\text { importantes como la posesión de conocimientos y las habilidades del candidato }\end{array}$ & \\
\hline Los procesos de selección se adaptan a cada puesto & \\
\hline $\begin{array}{l}\text { Sólo se selecciona a los candidatos que cuenten con la cualificación necesaria para } \\
\text { el puesto de trabajo }\end{array}$ & \\
\hline La inversión que la organización destina a la formación es considerable & \\
\hline $\begin{array}{l}\text { Se ofrece formación de distintos tipos (resolución de problemas, habilidades } \\
\text { técnicas...) }\end{array}$ & \\
\hline $\begin{array}{l}\text { Existen programas formales de formación para el desarrollo de habilidades } \\
\text { generales de los empleados }\end{array}$ & \\
\hline $\begin{array}{l}\text { Existen programas formales de formación para los trabajadores recién } \\
\text { incorporados para dotarles de las habilidades necesarias para su trabajo }\end{array}$ & \\
\hline Existen sistemas de remuneración que se basan en el rendimiento individual & \\
\hline $\begin{array}{l}\text { En las retribuciones a los empleados se toman en consideración sus habilidades y } \\
\text { conocimientos }\end{array}$ & \\
\hline Se cuenta con un sistema de evaluación del rendimiento (desempeño) & \\
\hline $\begin{array}{l}\text { Existen grupos formales de trabajo que trabajan de forma autónoma en cuestiones } \\
\text { de calidad, para el desarrollo de nuevos proyectos, en la resolución de conflictos, } \\
\text { de mejora, etc. }\end{array}$ & \\
\hline $\begin{array}{l}\text { Los trabajadores trabajan periódicamente en equipos de trabajo con cierta } \\
\text { autonomía }\end{array}$ & \\
\hline Se fomenta explícitamente el trabajo en equipo & \\
\hline $\begin{array}{l}\text { Los empleados, en general, conocen los objetivos y las estrategias de la } \\
\text { organización, salvo aquella información que por sus características puede resultar } \\
\text { en un riesgo estratégico }\end{array}$ & \\
\hline $\begin{array}{l}\text { Los trabajadores disponen de mecanismos tales como buzones de sugerencias, } \\
\text { banco de aportaciones u otro tipo de canales para plantear sus ideas, quejas y } \\
\text { aportaciones en general }\end{array}$ & \\
\hline $\begin{array}{l}\text { Se realizan encuestas o reuniones periódicas con los empleados para conocer su } \\
\text { grado de satisfacción }\end{array}$ & \\
\hline $\begin{array}{l}\text { Entre las personas que ocupan puestos inferiores y superiores de la estructura } \\
\text { organizativa existe una relación distante }\end{array}$ & \\
\hline La relación de los directivos con los empleados es espontánea e informal & \\
\hline Se favorece la participación de los empleados en las decisiones y acciones & \\
\hline Se fomenta la iniciativa y autonomía de los empleados en sus trabajos & \\
\hline Se percibe un clima de alta cooperación y confianza & \\
\hline Las diferencias de estatus* son altas & \\
\hline
\end{tabular}

*En el sentido de la posición que una persona ocupa en la organización. 